\title{
Checklist dos Copepoda Calanoida de água doce do Estado de São Paulo
}

\author{
Takako Matsumura-Tundisi, ${ }^{1,2}$ \& José Galizia Tundisi ${ }^{1}$ \\ ${ }^{1}$ Instituto Internacional de Ecologia, Rua Bento Carlos, 750, CEP 13560-660, São Carlos, SP, Brasil \\ ${ }^{2}$ Autor para correspondência: Takako Matsumura Tundisi, e-mail: takako@iie.com.br
}

MATSUMURA-TUNDISI, T. \& TUNDISI, J.G. Checklist of fresh-water Copepoda Calanoida from São Paulo State, Brazil. Biota Neotrop. 11(1a): http://www.biotaneotropica.org.br/v11n1a/en/abstract?inventory+ bn0251101a2011.

\begin{abstract}
Calanoida copepods from São Paulo State were studied extensively during the Project BIOTA/FAPESP (1999-2003). A number of 389 samples were collected from several hydric systems belonging at 22 UGRHI (Unit Management of Hydric Resources) of São Paulo State. Twelve (12) species of Calanoida were found, one of them a new species described as Notodiaptomus oliveirai (Matsumura-Tundisi et al. 2010) and registered in several water bodies of the UGRHI analysed except the Alto Tietê UGRHI. Other less frequent species however recorded already in São Paulo State such as Idiodiaptomus gracilipes, Trichodiaptomus coronatus, Notodiaptomus conifer, N. spinuliferus, N. transitans, N. isabelae, N. jatobensis and Pseudodiaptomus acutus were not found in the samplings of BIOTA/FAPESP.

Keywords: fresh-water Calanoida, biodiversity of the State of São Paulo, BIOTA/FAPESP Program.
\end{abstract}

MATSUMURA-TUNDISI, T. \& TUNDISI, J.G. Checklist dos Copepoda Calanoida de água doce do Estado de São Paulo, Brasil. Biota Neotrop. 11(1a): http://www.biotaneotropica.org.br/v11n1a/pt/abstract?inventory +bn0251101a2011.

Resumo: Copepoda Calanoida do Estado de São Paulo foi intensivamente estudado através da análise de 389 amostras obtidas dos corpos hidricos de 22 Unidades de Gerenciamento de Recursos Hidricos (UGRHI) do Estado de São Paulo durante o Programa BIOTA/FAPESP realizado entre 1999-2003. Foram identificadas 12 espécies sendo uma delas especie nova Notodiaptomus oliveirai foi encontrada em quase todas as bacias hidrográficas, exceto na UGRHI Alto Tietê. Uma outra espécie, provavelmente nova, necessita de um melhor estudo para a sua identificação. Algumas espécies de rara ocorrência já registradas no Estado de São Paulo tais como Idiodiaptomus gracilipes, Trichodiaptomus coronatus, Notodiaptomus conifer, N. spinuliferus, N. transitans, $N$. isabelae, $N$. jatobensis e Pseudodiaptomus acutus não foram encontradas nas amostragens do BIOTA/FAPESP. Palavras-chave: Calanoida de água doce, biota paulista, Programa BIOTA/FAPESP. 


\section{Introdução}

Os Copepoda Calanoida são microcrustáceos habitantes de águas estagnadas, fazendo parte da comunidade de zooplâncton de lagos, reservatórios e pequenas poças de água de existência transitória. Pertence ao Filo Arthropoda, Subfilo Crustacea, Classe Copepoda, Ordem Calanoida (Matsumura-Tundisi \& Da Silva 1999). Esse grupo tem grande importância no meio aquático, pois são organismos que contribuem com maior biomassa servindo de alimento para os organismos que ocupam níveis tróficos superiores tais como alevinos de peixes ou mesmo peixes planctôfagos. A maioria das espécies apresenta um grande endemismo sendo que sua ocorrência é bastante restrita a determinadas áreas e faixas latitudinais (Matsumura-Tundisi 1986). A maioria dos gêneros registrados no hemisfério Sul não ocorre no hemisfério Norte.

Duas famílias da Ordem Calanoida ocorrem em água doce: Pseudodiaptomidae e a família Diaptomidae.

Os primeiros estudos sobre Copepoda Calanoida do estado de São Paulo foram feitos por Sars (1901), que estudou os organismos pertencentes à família Diaptomidae. Todas as espécies descritas na América do Sul, foram, inicialmente, colocadas no gênero Diaptomus (Wright 1927). Posteriormente, as espécies com características comuns foram agrupadas, resultando em 11 gêneros (Brehm 1958) com os respectivos número de espécies: Odontodiaptomus: 3; Argyrodiaptoumus: 9; Rhacodiaptomus: 5; Aspinus: 1; Scolodiaptomus: 1; Calodiaptomus: 2; Dactylodiaptomus: 1; Idiodiaptomus: 1; Notodiaptomus: 23; Prionodiaptomus: 1; Trichodiaptomus: 1. No Brasil, alguns gêneros estão restritos à região Amazônica, como Rhacodiaptomus, Calodiaptomus e Aspinus. Entre os Notodiaptomus, algumas espécies ocorrem somente na região norte, outras na sudeste e outras mostram ampla distribuição, ocorrendo de norte a sul, como no caso do Argyrodiaptomus azevedoi.

Da família Pseudodiaptomidae duas espécies Pseudodiaptomus acutus Dahl, 1894, e Pseudodiaptomus richardi Dahl, 1894, ocorrem no Estado de São Paulo, na Baixada Santista, a primeira no Rio Baguaçu em Cananeia (Tundisi \& MatsumuraTundisi 1968, 2001) e a segunda na Represa Aguapeú (Progama BIOTA/FAPESP 2003).

\section{Metodologia}

Para elaboração da lista de espécies dos Copepoda Calanoida de água doce do Estado de São Paulo, foram consultadas as seguintes referências bibliográficas: Sars (1901), Van Douwe (1911), Brandorff (1976), Wright (1936a,1936b,1937), Tundisi \& Matsumura-Tundisi (1968), Tundisi (1980) USP/Dep. Ecologia (Universidade...1980), Inst. Pesca/Secr. Agr. (Instituto...1980), UFSCar/Grupo de Ecol. Aq. (Universidade...1980), Sendacz \& Kubo (1982), MatsumuraTundisi (1986), Matsumura-Tundisi \& Tundisi (2003), BIOTA/ FAPESP (Programa... 2003), Dussart \& Matsumura-Tundisi (1986), Matsumura-Tundisi (2008), Matsumura-Tundisi et al. (2010).

\section{Resultados e Discussão}

\section{Lista das espécies de Calanoida do estado São Paulo.}

A Tabela 1 lista as espécies de Calanoida registradas nos corpos de água do estado de São Paulo. A Tabela 2 lista os corpos de água analisados com as respectivas UGRHIs/bacias. A Figura 1 refere-se ao mapa do estado de São Paulo com as 22 Unidades de Gerenciamento de Recursos Hidricos (UGRHI) estabelecidas pela portaria do Governo do Estado de São Paulo no Plano Estadual de Recursos Hidricos (São Paulo 1994).
Algumas espécies como o Idiodiaptomus gracilipes descrita por Van Douwe (1911), através do material obtido do corpo de água do município de Itapura (SP), não teve mais registros posteriores pelos especialistas que trabalham com organismos zooplanctônicos. Outras espécies como Odontodiaptomus paulistanus, sua ocorrência encontra-se restrita à bacia do Alto Tietê (UGRHI-6 Alto Tietê) (Wright 1936b, Wright, 1938, Sendacz \& Kubo 1982, Programa BIOTA/FAPESP 2003). A ocorrência de Argyrodiaptomus azevedoi, já havia sido registrada na represa de Jupiá (bacia do Rio Paraná), no Projeto de Tipologia de reservatórios do Estado de São Paulo (Tundisi 1980) e mais recentemente no Programa Biota/FAPESP (Matsumura-Tundisi 2003), nos corpos de água das UGRHI-18 (São José dos Dourados) e UGRHI-19 (Baixo Tietê), todos sob a influência do Rio Paraná. Já a espécie Argyrodiaptomus furcatus ocorre nos corpos de água das bacias do Rio Grande, Paranapanema, Alto e Médio Tietê como foi constatada tanto no Projeto de Tipologia de reservatórios do Estado de São Paulo (Tundisi 1980) e também no Programa Biota/FAPESP (Matsumura-Tundisi 2003). Neste ultimo Projeto foi constatada a presença de A. furcatus também nos corpos de água da bacia do Mogi Guaçu (UGHI-9), Piracicaba/Jundiai/ Capivari (UGRHI-5), Tietê/Sorocaba (UGRHI-10).

As espécies mais amplamente distribuídas no Estado de São Paulo são Notodiaptomus iheringi e a nova espécie Notodiaptomus oliveirai descrita por Matsumura-Tundisi et al. (2010), ocorrendo em todos os corpos de água de quase todas as UGRHIs, exceto nas UGRHI-Alto Tietê, UGRHI-Mantiqueira, UGRHI-MogiGuaçu e UGRHI-Pardo, UGRHI-Baixada Santista para Notodiaptomus oliveirai, e UGRHIParaiba do Sul, UGRHI-Ribeira do Iguape e UGRHI-Pardo para Notodiaptomus iheringi.

Certas espécies tais como Notodiaptomus conifer, N. isabelae, $N$. jatobences, $N$. transitans que estavam presentes em corpos de água do estado de São Paulo, em 1979 (Tundisi 1980) não foram mais registradas no estudo feito em 2000 no Programa BIOTA/FAPESP (Matsumura-Tundisi 2003). Nos sistemas hídricos é comum a ocorrer mudanças na composição especifica dos organismos zooplanctônicos como foi observado por Matsumura-Tundisi \& Tundisi (2003) com relação ao grupo dos Copepoda Calanoida, nos corpos hídricos do estado de São Paulo. Na represa de Barra Bonita (SP) onde as espécies Argyrodiaptomus furcatus, Notodiaptomus conifer e Scolodiaptomus corderoi eram abundantes até a década de 1980, foram substituídos paulatinamente por Notodiaptomus iheringi, Notodiaptumus cearensis e Notodiaptumus oliverai n. spec. (Matsumura-Tundisi et al. 2010). Outras espécies tais como Notodiaptomus henseni, Notodiaptomus venezolanus deevoyorum foram registradas pela primeira vez no estado de São Paulo no Programa BIOTA/FAPESP.

\section{Comentários sobre a lista, riqueza do Estado comparado com outras regiões.}

O grupo dos Copepoda Calanoida foi muito bem estudada, além dos corpos de água do estado de São Paulo, também nos corpos de água da região amazônica abrangendo os estados do Amazonas, Pará, Acre e Rondônia, por Brandorff (1976, 1978), Dussart \& Robertson (1984), Andrade \& Brandorff (1975). A maioria das espécies que ocorrem na região amazônica são endêmicas da região diferindose das espécies de Calanoida que ocorrem em latitudes mais altas como as das regiões do sudeste e centro oeste do Brasil. Uma comparação sobre o numero de espécies da região amazônica com numero de espécies registrado no Estado de São Paulo verifica-se uma similaridade, ou seja, 21 espécies na região norte amazônica e 19 espécies na região sudeste, este número referente apenas ao do Estado de São Paulo. Assim, em virtude da escassez e da inconsistência do estudo da biodiversidade desses organismos nas 
Espécies de Copepoda Calanoida de água doce

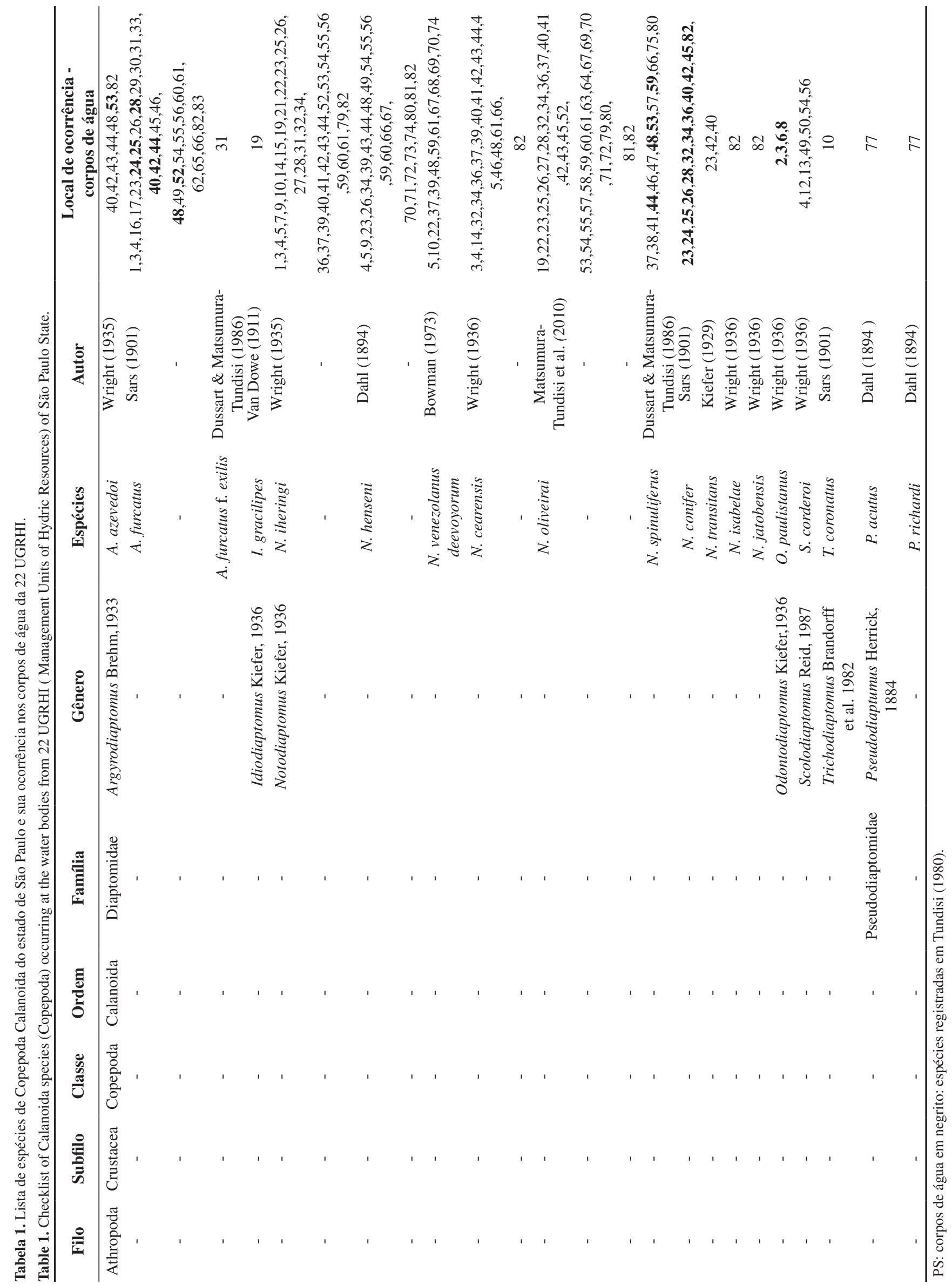


Tabela 2. Corpos de água com as coordenadas, nas 22 UGRHI do Estado de São Paulo.

Table 2. Water bodies studied in 22 UGRHI (Management Units of Hydric Resources) of Sao Paulo State with geographical coordinates.

\begin{tabular}{|c|c|c|c|c|}
\hline $\begin{array}{c}\text { Códigos } \\
\text { da UGRHI }\end{array}$ & UGRHI/Bacia & Corpos de água & $\begin{array}{c}\text { Códigos dos } \\
\text { corpos de água }\end{array}$ & Coordenadas \\
\hline \multirow[t]{11}{*}{6} & Alto Tietê & Represa Billings & 1 & $23^{\circ} 45^{\prime} 89^{\prime \prime} \mathrm{S}-46^{\circ} 30^{\prime} 96^{\prime \prime} \mathrm{W}$ \\
\hline & - & Billings-Riacho Grande & 2 & $23^{\circ} 45^{\prime} 89^{\prime \prime} \mathrm{S}-46^{\circ} 30^{\prime} 96^{\prime \prime} \mathrm{W}$ \\
\hline & - & Represa Paiva Castro & 3 & $23^{\circ} 19^{\prime} 95^{\prime \prime} \mathrm{S}-46^{\circ} 39^{\prime} 24^{\prime \prime} \mathrm{W}$ \\
\hline & - & Represa de Águas Claras & 4 & $23^{\circ} 23^{\prime} 91^{\prime \prime} \mathrm{S}-46^{\circ} 39^{\prime} 52^{\prime \prime} \mathrm{W}$ \\
\hline & - & Represa Taiaçupeba & 5 & $23^{\circ} 34^{\prime} 80^{\prime \prime} \mathrm{S}-46^{\circ} 16^{\prime} 92^{\prime \prime} \mathrm{W}$ \\
\hline & - & Represa Ribeirão do Campo & 6 & $23^{\circ} 39^{\prime} 76^{\prime \prime} \mathrm{S}-46^{\circ} 49^{\prime} 54^{\prime \prime} \mathrm{W}$ \\
\hline & - & Lago do Parque Ecológico & 7 & $22^{\circ} 29^{\prime} 07^{\prime \prime} \mathrm{S}-46^{\circ} 31^{\prime} 08^{\prime \prime} \mathrm{W}$ \\
\hline & - & Represa Ponte Nova & 8 & $23^{\circ} 35^{\prime} 83^{\prime \prime} \mathrm{S}-45^{\circ} 56^{\prime} 78^{\prime \prime} \mathrm{W}$ \\
\hline & - & Represa Jundiai & 9 & $23^{\circ} 39^{\prime} 01^{\prime \prime} \mathrm{S}-46^{\circ} 11^{\prime} 51^{\prime \prime} \mathrm{W}$ \\
\hline & - & Represa de Guarapiranga & 10 & $23^{\circ} 40^{\prime} 48^{\prime \prime} \mathrm{S}-46^{\circ} 43^{\prime} 36^{\prime \prime} \mathrm{W}$ \\
\hline & - & Represa de Juqueri & 11 & - \\
\hline \multirow[t]{2}{*}{1} & Mantiqueira & Lago das ninfeas & 12 & $22^{\circ} 41^{\prime} 44^{\prime \prime} \mathrm{S}-45^{\circ} 29^{\prime} 12^{\prime \prime} \mathrm{W}$ \\
\hline & - & Lago do Horto & 13 & $22^{\circ} 41^{\prime} 48^{\prime \prime} \mathrm{S}-45^{\circ} 28^{\prime} 80^{\prime \prime} \mathrm{W}$ \\
\hline \multirow[t]{5}{*}{9} & Mogi Guaçu & Represa MogiGuaçu & 14 & $22^{\circ} 22^{\prime} 93^{\prime \prime} \mathrm{S}-46^{\circ} 53^{\prime} 93^{\prime \prime} \mathrm{W}$ \\
\hline & - & Lago da Churrascaria Sto. Antonio & 15 & $22^{\circ} 33^{\prime} 86^{\prime \prime} \mathrm{S}-46^{\circ} 32^{\prime} 31^{\prime \prime} \mathrm{W}$ \\
\hline & - & Lago as Fazenda Aurora & 16 & $20^{\circ} 59^{\prime} 82^{\prime \prime} \mathrm{S}-47^{\circ} 58^{\prime} 95^{\prime \prime} \mathrm{W}$ \\
\hline & - & Represa do CEPTA( Barrinha) & 17 & $25^{\circ} 08^{\prime} 55^{\prime \prime} \mathrm{S}-52^{\circ} 01^{\prime} 52^{\prime \prime} \mathrm{W}$ \\
\hline & - & Lago Sta. Cruz da Conceição & 18 & $19^{\circ} 59^{\prime} 50^{\prime \prime} \mathrm{S}-49^{\circ} 23^{\prime} 90^{\prime \prime} \mathrm{W}$ \\
\hline \multirow[t]{3}{*}{22} & Pontal do Paranapanema & Represa Rosana & 19 & $22^{\circ} 35^{\prime} 40^{\prime \prime} \mathrm{S}-52^{\circ} 51^{\prime} 14^{\prime \prime} \mathrm{W}$ \\
\hline & - & Represa Tavares & 21 & - \\
\hline & - & Represa Taquaruçu & 22 & $22^{\circ} 35^{\prime} 40^{\prime \prime} \mathrm{S}-52^{\circ} 51^{\prime} 14^{\prime \prime} \mathrm{W}$ \\
\hline \multirow[t]{6}{*}{14} & Alto Paranapanema & Represa Capivara & 23 & $22^{\circ} 54^{\prime} 01^{\prime \prime} \mathrm{S}-40^{\circ} 47^{\prime} 30^{\prime \prime} \mathrm{W}$ \\
\hline & - & Represa de Piraju & 24 & - \\
\hline & - & Rio Pari & 88 & - \\
\hline & - & Represa Batista & 87 & - \\
\hline & - & Represa Jurumirim & 25 & $23^{\circ} 13^{\prime} 38^{\prime \prime} \mathrm{S}-49^{\circ} 13^{\prime} 31^{\prime \prime} \mathrm{W}$ \\
\hline & - & Represa Chavantes & 26 & $23^{\circ} 08^{\prime} 27^{\prime \prime} \mathrm{S}-49^{\circ} 42^{\prime} 24^{\prime \prime} \mathrm{W}$ \\
\hline \multirow[t]{2}{*}{17} & Medio Paranapanema & Represa Canoa 1 & 27 & $22^{\circ} 56^{\prime} 31^{\prime \prime} \mathrm{S}-50^{\circ} 30^{\prime} 41^{\prime \prime} \mathrm{W}$ \\
\hline & - & Represa Salto Grande & 28 & $22^{\circ} 53^{\prime} 53^{\prime \prime} \mathrm{S}-49^{\circ} 59^{\prime} 32^{\prime \prime} \mathrm{W}$ \\
\hline \multirow[t]{8}{*}{13} & Tietê/Jacaré & Represa do Chile & 29 & $21^{\circ} 52^{\prime} 02^{\prime \prime} \mathrm{S}-47^{\circ} 51^{\prime} 92^{\prime \prime} \mathrm{W}$ \\
\hline & - & Represa Jacaré Pepira & 30 & - \\
\hline & - & Represa do Lobo/Broa & 31 & $22^{\circ} 10^{\prime} 22^{\prime \prime} \mathrm{S}-47^{\circ} 54^{\prime} 24^{\prime \prime} \mathrm{W}$ \\
\hline & - & Represa de Bariri & 32 & $22^{\circ} 09^{\prime} 73^{\prime \prime} \mathrm{S}-48^{\circ} 44^{\prime} 56^{\prime \prime} \mathrm{W}$ \\
\hline & - & Represa do Clube Nautico & 33 & $21^{\circ} 42^{\prime} 40^{\prime \prime} \mathrm{S}-48^{\circ} 01^{\prime} 61^{\prime \prime} \mathrm{W}$ \\
\hline & - & Represa Ibitinga & 34 & $21^{\circ} 45^{\prime} 41^{\prime \prime} \mathrm{S}-48^{\circ} 58^{\prime} 47^{\prime \prime} \mathrm{W}$ \\
\hline & - & Represa 29 & 35 & $21^{\circ} 53^{\prime} 74^{\prime \prime} \mathrm{S}-47^{\circ} 49^{\prime} 03^{\prime \prime} \mathrm{W}$ \\
\hline & - & Represa Barra Bonita & 36 & $22^{\circ} 31^{\prime} 82^{\prime \prime} \mathrm{S}-48^{\circ} 31^{\prime} 23^{\prime \prime} \mathrm{W}$ \\
\hline \multirow[t]{5}{*}{16} & Tietê/Batalha & lago laranja azeda & 37 & $21^{\circ} 50^{\prime} 49^{\prime \prime} \mathrm{S}-48^{\circ} 57^{\prime} 23^{\prime \prime} \mathrm{W}$ \\
\hline & - & Represa de Ibitinga & 38 & $21^{\circ} 50^{\prime} 49^{\prime \prime} \mathrm{S}-48^{\circ} 57^{\prime} 23^{\prime \prime} \mathrm{W}$ \\
\hline & - & Represa de Bariri & 39 & $21^{\circ} 39^{\prime} 31^{\prime \prime} \mathrm{S}-49^{\circ} 08^{\prime} 48^{\prime \prime} \mathrm{W}$ \\
\hline & - & Represa de Promissão & 40 & $21^{\circ} 20^{\prime} 33^{\prime \prime} \mathrm{S}-49^{\circ} 44^{\prime} 37^{\prime \prime} \mathrm{W}$ \\
\hline & - & Promissão/Pongai & 41 & $21^{\circ} 41^{\prime} 22^{\prime \prime} \mathrm{S}-49^{\circ} 18^{\prime} 30^{\prime \prime} \mathrm{W}$ \\
\hline \multirow[t]{3}{*}{19} & Baixo Tietê & Represa Nova Avanhandava & 42 & $21^{\circ} 17^{\prime} 42^{\prime \prime} \mathrm{S}-50^{\circ} 08^{\prime} 17^{\prime \prime} \mathrm{W}$ \\
\hline & - & Represa Três Irmãos & 43 & $20^{\circ} 54^{\prime} 02^{\prime \prime} \mathrm{S}-50^{\circ} 34^{\prime} 03^{\prime \prime} \mathrm{W}$ \\
\hline & - & Represa Jupiá & 44 & $20^{\circ} 45^{\prime} 09^{\prime \prime} \mathrm{S}-51^{\circ} 37^{\prime} 21^{\prime \prime} \mathrm{W}$ \\
\hline \multirow[t]{3}{*}{10} & Tietê/Sorocaba & Represa de Itupareranga & 45 & $23^{\circ} 35^{\prime} 81^{\prime \prime} \mathrm{S}-47^{\circ}$ ' 8 ' $07^{\prime \prime} \mathrm{W}$ \\
\hline & - & Represa Prainha & 46 & $23^{\circ} 34^{\prime} 89^{\prime \prime} \mathrm{S}-4^{\circ} 26^{\prime} 04^{\prime \prime} \mathrm{W}$ \\
\hline & - & Represa Heldberg & 47 & $23^{\circ} 25^{\prime} 61^{\prime \prime} \mathrm{S}-47^{\circ} 35^{\prime} 68^{\prime \prime} \mathrm{W}$ \\
\hline 18 & São José dos Dourados & Represa de Ilha Solteira & 48 & $20^{\circ} 22^{\prime} 26^{\prime \prime} \mathrm{S}-51^{\circ} 20^{\prime} 06^{\prime \prime} \mathrm{W}$ \\
\hline 4 & Pardo & Represa Graminha & 49 & $21^{\circ} 34^{\prime} 81^{\prime \prime} \mathrm{S}-47^{\circ} 37^{\prime} 16^{\prime \prime} \mathrm{W}$ \\
\hline
\end{tabular}


Tabela 2. Continuação...

Table 2. Continued...

\begin{tabular}{|c|c|c|c|c|}
\hline $\begin{array}{c}\text { Códigos } \\
\text { da UGRHI }\end{array}$ & UGRHI/Bacia & Corpos de água & $\begin{array}{c}\text { Códigos dos } \\
\text { corpos de água }\end{array}$ & Coordenadas \\
\hline & - & Represa do Limoeiro & 50 & - \\
\hline & - & Represa de Euclides da Cunha & 51 & - \\
\hline \multirow[t]{2}{*}{12} & Baixo Pardo/R. Grande & Represa de Porto Colombia & 52 & $20^{\circ} 07^{\prime} 35^{\prime \prime} \mathrm{S}-48^{\circ} 33^{\prime} 43^{\prime \prime} \mathrm{W}$ \\
\hline & - & Represa de Morimbondo & 53 & $20^{\circ} 31^{\prime} 02^{\prime \prime} \mathrm{S}-48^{\circ} 53^{\prime} 06^{\prime \prime} \mathrm{W}$ \\
\hline \multirow[t]{4}{*}{8} & Sapucai/R.Grande & Represa de Jaguara & 54 & $20^{\circ} 04^{\prime} 75^{\prime \prime} \mathrm{S}-47^{\circ} 24^{\prime} 61^{\prime \prime} \mathrm{W}$ \\
\hline & - & Represa Estreito & 84 & - \\
\hline & - & Represa Igarapava & 55 & $20^{\circ} 59^{\prime} 28^{\prime \prime} \mathrm{S}-47^{\circ} 43^{\prime} 93^{\prime \prime} \mathrm{W}$ \\
\hline & - & Represa de Volta Grande & 56 & $20^{\circ} 08^{\prime} 67^{\prime \prime} \mathrm{S}-48^{\circ} 02^{\prime} 34^{\prime \prime} \mathrm{W}$ \\
\hline \multirow[t]{3}{*}{15} & Turvo/ R.Grande & Represa Cestari & 57 & $21^{\circ} 15^{\prime} 68^{\prime \prime} \mathrm{S}-48^{\circ} 21^{\prime} 29^{\prime \prime} \mathrm{W}$ \\
\hline & - & Represa Sant'Ana \& Sto. Antonio & 58 & $21^{\circ} 05^{\prime} 38^{\prime \prime} \mathrm{S}-49^{\circ} 01^{\prime} 21^{\prime \prime} \mathrm{W}$ \\
\hline & - & Represa de Água Vermelha & 59 & $19^{\circ} 56^{\prime} 57^{\prime \prime} \mathrm{S}-49^{\circ} 40^{\prime} 30^{\prime \prime} \mathrm{W}$ \\
\hline \multirow[t]{7}{*}{5} & Piracicaba/Jundiai/Capivari & Represa Paramirim & 60 & - \\
\hline & - & Represa Igaratá & 61 & $23^{\circ} 11^{\prime} 41^{\prime \prime} \mathrm{S}-46^{\circ} 07^{\prime} 24 \mathrm{~W}$ \\
\hline & - & Represa Piracaia & 62 & $23^{\circ} 04^{\prime} 24^{\prime \prime} \mathrm{S}-46^{\circ} 19^{\prime} 32^{\prime \prime} \mathrm{W}$ \\
\hline & - & Represa Cachoeira & 63 & $23^{\circ} 07^{\prime} 52^{\prime \prime} \mathrm{S}-46^{\circ} 17^{\prime} 31^{\prime \prime} \mathrm{W}$ \\
\hline & - & Lagoa Pousada C.A. & 64 & - \\
\hline & - & Represa Atibaia & 65 & $23^{\circ} 05^{\prime} 49^{\prime \prime} \mathrm{S}-46^{\circ} 38^{\prime} 13^{\prime \prime} \mathrm{W}$ \\
\hline & - & Represa Atibainha & 66 & $23^{\circ} 10^{\prime} 55^{\prime \prime} \mathrm{S}-46^{\circ} 23^{\prime} 20^{\prime \prime} \mathrm{W}$ \\
\hline \multirow[t]{4}{*}{2} & Paraiba do Sul & Represa de Paraibuna & 67 & $23^{\circ} 23^{\prime} 11^{\prime \prime} \mathrm{S}-45^{\circ} 26^{\prime} 30^{\prime \prime} \mathrm{W}$ \\
\hline & - & Represa Santa Branca & 68 & $23^{\circ} 22^{\prime} 05^{\prime \prime} \mathrm{S}-45^{\circ} 51^{\prime} 28^{\prime \prime} \mathrm{W}$ \\
\hline & - & Represa Itapeva & 85 & - \\
\hline & - & Represa Funil & 86 & - \\
\hline \multirow[t]{7}{*}{11} & Ribeira do Iguape & Represa orto Raso & 69 & $24^{\circ} 03^{\prime} 30^{\prime \prime} \mathrm{S}-47^{\circ} 24^{\prime} 35^{\prime \prime} \mathrm{W}$ \\
\hline & - & Represa Serraria & 70 & $24^{\circ} 08^{\prime} 43^{\prime \prime} \mathrm{S}-47^{\circ} 32^{\prime} 28^{\prime \prime} \mathrm{W}$ \\
\hline & - & Represa Alecrim & 71 & $24^{\circ} 04^{\prime} 46^{\prime \prime} \mathrm{S}-47^{\circ} 28^{\prime} 34^{\prime \prime} \mathrm{W}$ \\
\hline & - & Represa Cachoeira da França & 72 & $23^{\circ} 56^{\prime} 04^{\prime \prime} \mathrm{S}-47^{\circ} 11^{\prime} 21^{\prime \prime} \mathrm{W}$ \\
\hline & - & Represa Fumaça & 73 & $24^{\circ} 00^{\prime} 16^{\prime \prime} \mathrm{S}-47^{\circ} 05^{\prime} 44^{\prime \prime} \mathrm{W}$ \\
\hline & - & Represa da Barra & 74 & $24^{\circ} 00^{\prime} 60^{\prime \prime} \mathrm{S}-47^{\circ} 21^{\prime} 28^{\prime \prime} \mathrm{W}$ \\
\hline & - & Represa Jurupara & 75 & $23^{\circ} 57^{\prime} 19^{\prime \prime} \mathrm{S}-47^{\circ} 23^{\prime} 58^{\prime \prime} \mathrm{W}$ \\
\hline \multirow[t]{2}{*}{7} & Baixada Santista & Represa Aguapeú- Itanhaem & 77 & $24^{\circ} 06^{\prime} 52^{\prime \prime} \mathrm{S}-46^{\circ} 47^{\prime} 19^{\prime \prime} \mathrm{W}$ \\
\hline & - & Represa rio das Pedras/Cubatão & 78 & $23^{\circ} 51^{\prime} 25^{\prime \prime} \mathrm{S}-46^{\circ} 27^{\prime} 52^{\prime \prime} \mathrm{W}$ \\
\hline \multirow[t]{5}{*}{20} & Aguapei & Represa Sete de Setembro (Tupã) & 79 & $21^{\circ} 51^{\prime} 44^{\prime \prime} \mathrm{S}-50^{\circ} 32^{\prime} 10^{\prime \prime} \mathrm{W}$ \\
\hline & - & Rio Aguapei & 80 & $21^{\circ} 42^{\prime} 24^{\prime \prime} \mathrm{S}-50^{\circ} 30^{\prime} 48^{\prime \prime} \mathrm{W}$ \\
\hline & - & Lago Rinopolis & 81 & $21^{\circ} 41^{\prime} 01^{\prime \prime} \mathrm{S}-50^{\circ} 36^{\prime} 35^{\prime \prime} \mathrm{W}$ \\
\hline & - & Rio Parana- Lagoas marginais & 82 & $21^{\circ} 20^{\prime} 53^{\prime \prime} \mathrm{S}-51^{\circ} 51^{\prime} 45^{\prime \prime} \mathrm{W}$ \\
\hline & - & Lagoa Marreco & 83 & $21^{\circ} 06^{\prime} 04^{\prime \prime} \mathrm{S}-51^{\circ} 43^{\prime} 53^{\prime \prime} \mathrm{W}$ \\
\hline 21 & Peixe & - & - & - \\
\hline
\end{tabular}

diversas regiões do Brasil é difícil fazer qualquer referência quanto à riqueza de espécies desse grupo taxonômico nas várias regiões do Brasil em termos comparativos.

\section{Principais avanços relacionados ao Programa BIOTA}

Apesar de que o grupo dos Copepoda Calanoida no Estado de São Paulo ter sido bem estudado por pesquisadores especializados em zooplancton, o Programa BIOTA deu um avanço muito grande, pois permitiu a formação de grupos de pesquisa mais especializados em grupos taxonômicos do zooplancton, (Protozoa, Rotifera, Cladocera, Copepoda-Calanoida e Copepoda Cyclopoida) permitindo conhecer e explorar de forma detalhada e precisa a identificação dos organismos e também a sua distribuição, abundância e frequiência de ocorrência nos corpos de água do Estado de São Paulo. Esses conhecimentos somente foram possíveis de se obter, pois o BIOTA possibilitou a exploração de 250 corpos de água inseridos nas 22 Unidades de Gerenciamento de Recursos Hídricos (UGRHI) e utilizar o sistema SINBIOTA, desenvolvido por outro grupo de pesquisa (Joly 1998) e que permitiu o mapeamento da ocorrência e distribuição das espécies de Calanoida no Estado de São Paulo.

\section{Principais grupos de pesquisa}

Grupos de Pesquisa em "Biodiversidade de zooplancton" com especialistas em Protozoa, Rotifera, Cladocera e Copepoda que dê continuidade ao trabalho que foi feito no BIOTA, não existem, pois os docentes das Universidades tem outros compromissos e ao 


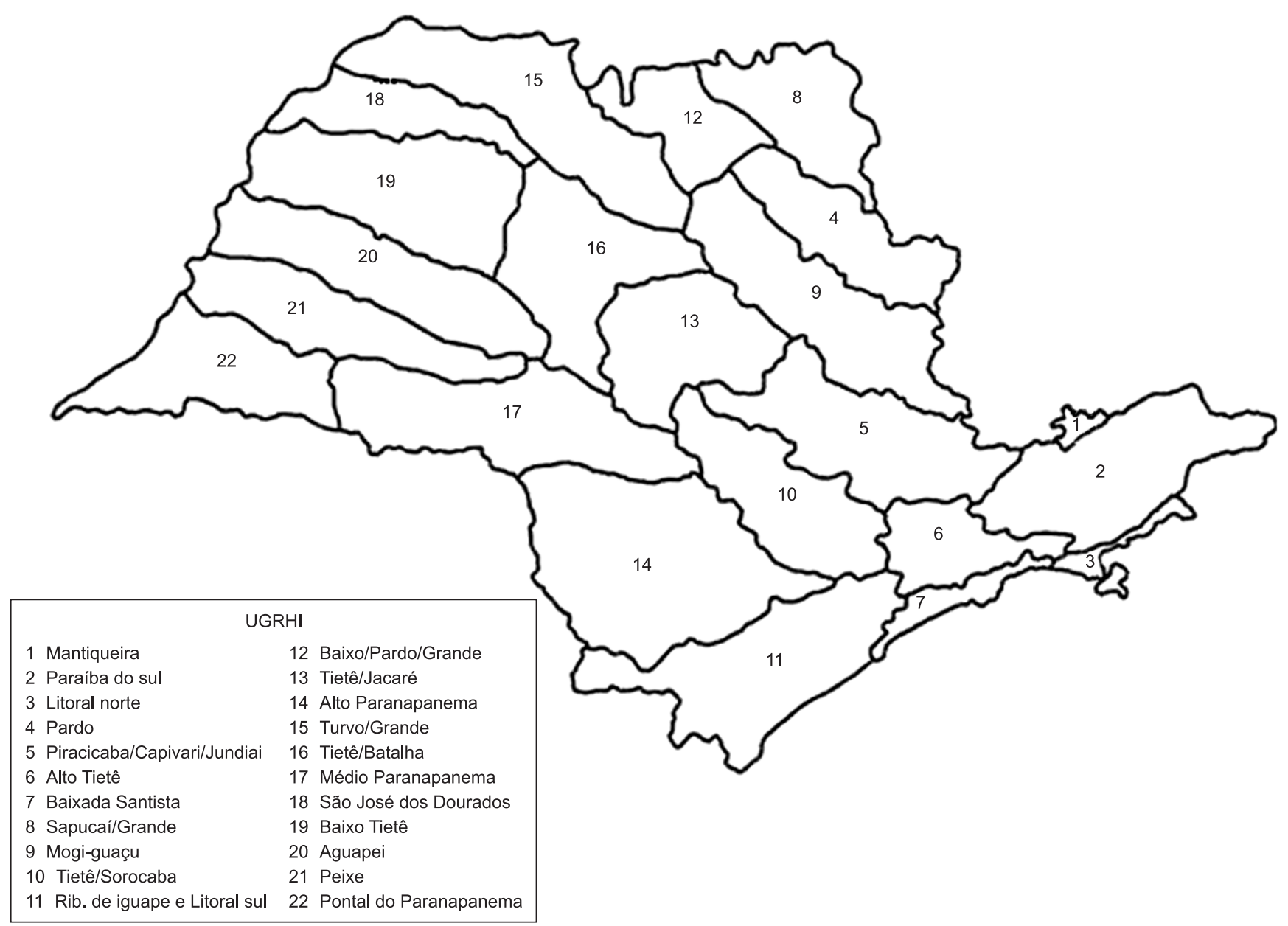

Figura 1. Legenda em português: Mapa do Estado de São Paulo com as 22 Unidades de Gerenciamento de Recursos Hídricos, onde foram estudados os corpos de água (média de 10 amostragens em cada UGRHI).

Figure 1. Legenda em inglês: Map of São Paulo State with 22 UGRHI (Management Units of Hydric Resources), where 220 water bodies were sampled.

finalizar o Programa BIOTA/FAPESP não deram mais continuidade inclusive com os estudantes. Os bolsistas de mestrado, doutorado, pós-doutorado que obtiveram bolsas da FAPESP, não conseguiram também dar continuidade à pesquisa, pois não tiveram oportunidade de se enquadrar nas Instituições de pesquisa em Biodiversidade.

\section{Principais acervos}

Acervos referentes à Biodiversidade de zooplancton têm-se as coleções de amostras de zooplancton obtidas dos corpos de água das 22 UGRHI do estado de São Paulo e que se encontram cadastradas no museu de plâncton do Instituto Internacional de Ecologia de São Carlos e no museu de plâncton da Universidade Federal de São Carlos (Departamento de Ecologia e Biologia Evolutiva). São 390 amostras de plâncton fixadas e preservadas em solução de formol a $4 \%$. Exemplares de holótipo e parátipos da espécie nova de Calanoida (Notodiaptomus oliveirai) estão depositados nas coleções do DCBU (Departamento de Ciências Biológicas da Universidade Federal de São Carlos).

\section{Principais lacunas do conhecimento}

As principais lacunas do conhecimento sobre o grupo taxonômico Copepoda Calanoida são a falta de informações sobre ocorrência de espécies em corpos de água de outras bacias hidrográficas e que pertencem a outros estados do Brasil. Seria de importância fundamental implantar em cada Estado, um programa de BIOTA para poder explorar com maior profundidade, os organismos aquáticos formando grupos de pesquisa em biodiversidade aquática.

\section{Perspectivas de pesquisa em Copepoda Calanoida para os próximos 10 anos}

Perspectivas de pesquisa com relação ao grupo dos Copepoda Calanoida para os próximos 10 anos são quase nulas, enquanto não houver uma política de incentivo aos jovens pesquisadores (doutorados e pós doutorados), de ingressarem em Instituições de Pesquisa (Não Universidades) que tenham suporte de Órgãos Públicos para pesquisa em biodiversidade.

\section{Referências bibliográficas}

ANDRADE, E.R. \& BRANDORFF, G.O. 1975. Uma nova espécie de Diaptomidae (Crustacea, Copepoda) "Diaptomus" negrensis das águas pretas perto de Manaus. Acta Amazonica 5(1):97-103.

BRANDORFF, G.O. 1976. The geographic distribution of the Diaptomidae in South America (Crustacea,Copepoda). Rev. Brasil. Biol. 36(3):613-627. 
BRANDORFF, G.O. 1978. Ein neuer Diaptomidae (Crustacea, Copepoda) "Diaptomus" ohlei aus dem brasilianischen Amazonasgebiet. Arch. Hydrobiol. 82 (1-4):295-299.

BREHM, V. Von. 1958. Bemerkungen zu einigen Kopepoden Südamerikas. Sitzungsberichten der öst. Akad. Wiss. Abt. I 167:139-171.

DUSSART, B. \& ROBERTSON, B. 1984. Notodiaptomus paraensis $\mathrm{n}$. sp. a new diaptomid (Crustacea, Copepoda) from the Brazialian Amazon. Amazoniana 8(3):389-394.

DUSSART, B.H. \& MATSUMURA-TUNDISI, T. 1986. Nouvelles espèces de Calanoïdes du Brèsil. Rev. Brasil.Biol. 46(1):249-255.

JOLY, C.A. 1998 Strengthening of the BIOTA/FAPESP information system and study of the development of a GIS for the program (A description of the SINBIOTA/SP information system and activities). PROJETO TEMATICO/FAPESP - Biota-FAPESP: www.biota.org.br

MATSUMURA-TUNDISI, T. 1986. Latitudinal distribution of Calanoida copepods in freshwater aquatic systems of Brazil. Rev. Brasil.Biol. 46(3):527-553.

MATSUMURA-TUNDISI, T. \& TUNDISI, J.G. 2003.Calanoida (Copepoda) species composition changes in the reservoirs of São Paulo State (Brazil in the last twenty years. Hydrobiologia 504:215-222. http://dx.doi. org/10.1023/B:HYDR.0000008521.43711.35

MATSUMURA-TUNDISI, T. 2008. Rectification of description of Notodiaptomus spinuliferus Dussart and Matsumura-Tundisi. Braz.J. Biol. Vol.68(3): 683-684.

MATSUMURA-TUNDISI, T., ESPINDOLA, E.L.G., TUNDISI, J.G., SOUZA-SOARES, F. \& DEGANI, R.M. 2010. A new species of Notodiaptomus Kiefer (Crustacea, Copepoda, Calanoida, Diaptomidae) from Brazil. Braz. J. Biol. 70(3)867-870. (Suppl.). http://dx.doi. org/10.1590/S1519-69842010000400017

PROGRAMA BIOTA/FAPESP. 2003. Biodiversidade do zooplancton de águas continentais. Progress Report, v.2,3,4. (Processo: 1998/05091-2).

REID, J.W. 1987. Scolodiaptomus, a new genus proposed for Diaptomus (sensu lato) corderoi Wright, and description of Notodiaptomus brandorfii, new species (Copepoda:Calanoida), from Brazil. J. Crust. Biol. 7(2):364379. http://dx.doi.org/10.2307/1548616
SÃO PAULO (Estado). 1994. Lei n. 9034 (27/12/94) - UGRHIs - Plano Estadual de Recursos Hidricos.

SARS, G.O. 1901. Contribution to the knowledge of the entomostraca of South America. Part 2. Copepoda-Ostracoda. Arch. Math. Mater. 24:1-46.

SENDACZ, S. \& KUBO, E. 1982. Copepoda (Calanoida e Cyclopoida) de reservatórios do Estado de São Paulo. B. Inst. Pesca 9:51-89.

TUNDISI, J.G. 1980. Tipologia de reservatórios do Estado de São Paulo. Progress Report vol. 1,2,3. (FAPESP- Processo: 1290-7/78).

TUNDISI, J.G. \& MATSUMURA-TUNDISI, T. 1968. Plankton studies in a mangrove environment. V. Salinity tolerances of some planktonic crustaceans. Bol. Inst. Oceanogr. S.paulo 17(1):57-65.

TUNDISI, J.G. \& MATSUMURA-TUNDISI, T. 2001. The lagoon region and estuary ecosystem of Cananeia, Brazil. In Ecological studies - coastal marine ecosystems in Latin America (U. Seeliger \& Kjerfve, ed.) Springer-Verlag Berlin Heidelberg, v.144, p.119-130.

VAN DOWE, C. 1911. Neue Suswasser-Copepoden aus Brasilien. Zool. Anzeiger. 37:161-163.

WRIGHT, S. 1927. A revision of the South American species of Diaptomus. Trans. Amer. Microsc. Soc. 46(2):73-103, plates: I-IX.

WRIGHT, S. 1935. Three new species of Diaptomus from Northeast Brazil. Ann. Acad. Brasil. Sci. 7(3):213-233, 4 pl.

WRIGHT, S. 1936a. A revision of the South American species of Pseudodiaptomus Ann. da Acad. Brasileira de Sciencias, tomo VIII, n.1, p.1-22, plates I,II,III.

WRIGHT, S. 1936b. Preliminary report on six new species of Diaptomus from Brazil. Ann. da Acad. Brasileira de Sciencias, tomo VIII, n.2, p.79-84, plates I, II.

WRIGHT, S. 1937. A review of some species of Diaptomus from São Paulo. Ann. Acad. Brasil. Sci., tomo IX, n.1, p.65-80, plates I, II,III.

WRIGHT, S., 1938. A review of the Diaptomus bergi group, with descriptions of two new species. Trans. Amer. Microsc. Soc. 57:297-315. http://dx.doi. org/10.2307/3222699 